\title{
Comment on: 'Trends in the lifetime risk of developing cancer in great Britain: comparison of risk for those born from 1930 to 1960'-cancer predictions need more context
}

JL Oke ${ }^{\star}, 1, B D$ Nicholson ${ }^{1}$ and B Shinkins ${ }^{1}$

${ }^{1}$ Nuffield Department of Primary Care Health Sciences, University of Oxford, Radcliffe Observatory Quarter, Woodstock Road, Oxford OX2 6GG, UK

Sir,

Projections of lifetime risk and cancer incidence for the next 25 years reported by Ahmad et al (2015) are alarming but probably realistic. In the last 30 years, the incidence of all cancers in the United Kingdom has risen from 293 cases per 100000 persons in 1975 to 396 per 100000 in 2011 (Cancer Research UK, 2012), a rise of $35 \%$. We were, however, surprised to such limited discussion or analysis of cancer mortality trends over the equivalent time period, which has fallen 21\% since 1971 (Cancer Research UK, 2012).

There has been a steady and linear increase over time in cancer incidence (Figure 1, solid black line). Extrapolating this trend forward (black dotted line) using simple linear regression produces incidence rates that generate lifetime and cumulative risks that are broadly in line with Ahmad et al's more

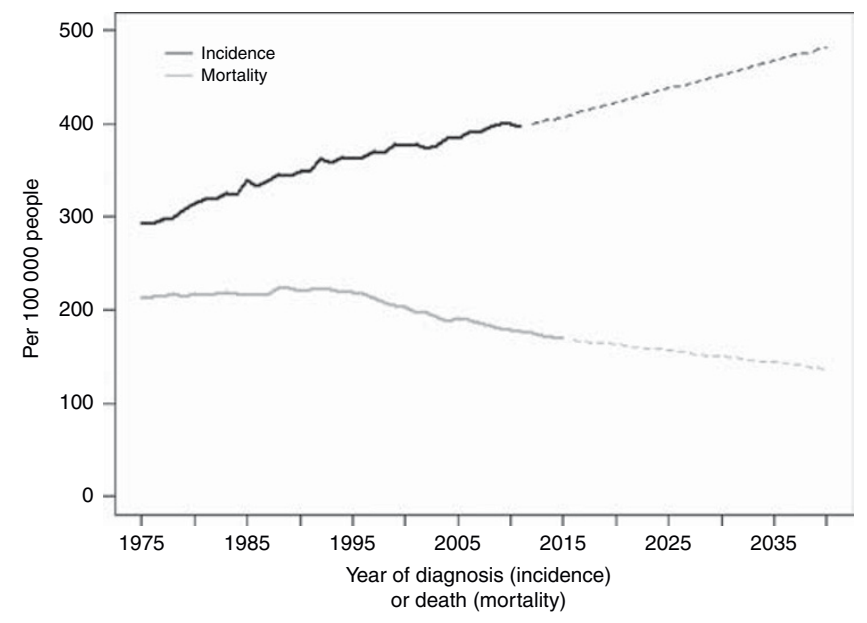

Figure 1. All-cancer incidence and mortality in the United Kingdom from 1975 to 2011(solid lines) and projected incidence and mortality (dashed lines). sophisticated approach. Using the same method to extrapolate the trend in allcancer mortality (solid grey line) suggests that all-cancer mortality will continue to decline (grey dashed line). In short, extrapolating current trends forward sends incidence and mortality in different directions, and this suggests a future in which cancer becomes more common but at the same time more benign.

One explanation for detecting increasing levels of cancer on the scale suggested by Ahmad et al without a concomitant increase in mortality is the detection of disease that will not go on to cause symptoms or death: 'overdiagnosis' (Welch and Black, 2010). This was acknowledged as contributory to the increased incidence in prostate cancer in relation to PSA testing, yet similar trends can be seen for thyroid, kidney, melanoma and breast cancer. Although it is methodologically challenging to take into account the impact of over-diagnosis and its underlying causes, diagnostic drift, increasing test sensitivity and changing competing mortality risks, these are important considerations to note when interpreting incidence data (Carter et al, 2015).

We call on the authors to publish their projected mortality rates to provide greater context to these worrying figures. The public deserve clear information about the drivers behind them, especially given the cumulative risk of overdiagnosis in an ageing population.

\section{CONFLICT OF INTEREST}

The authors declare no conflict of interest.

\section{REFERENCES}

Ahmad AS, Ormiston-Smith N, Sasieni PD (2015) Trends in the lifetime risk of developing cancer in Great Britain: comparison of risk for those born from 1930 to 1960. Br J Cancer 112: 943-947.

Cancer Research UK (2012) Cancer incidence for all cancers combined. Available from http://www.cancerresearchuk.org/cancer-info/cancerstats/incidence/ all-cancers-combined/\#UK (accessed on 13 February 2015).

Cancer Research UK (2012) Cancer mortality for all cancers combined. Available from http://www.cancerresearchuk.org/cancer-info/cancerstats/mortality/ all-cancers-combined (accessed on 13 February 2015).

Carter JL, Coletti RJ, Harris RP (2015) Quantifying and monitoring overdiagnosis in cancer screening: a systematic review of methods. BMJ 350: g7773.

Welch HG, Black WC (2010) Overdiagnosis in cancer. J Natl Cancer Inst 102(9): 605-613.

*Correspondence: Dr J Oke; E-mail: Jason.oke@phc.ox.ac.uk

Published online 23 June 2015

(c) 2015 Cancer Research UK. All rights reserved 0007-0920/15

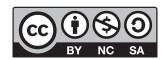

http://creativecommons.org/licenses/by-nc-sa/4.0/
$\mathrm{BIC}$ OPEN 0

\section{Comment on 'Dexamethasone exerts profound immunologic interference on treatment efficacy for} recurrent glioblastoma'

Susannah Ellsworth ${ }^{*}$ and Stuart A Grossman ${ }^{2}$

${ }^{1}$ Department of Radiation Oncology, Indiana University School of Medicine, Indianapolis, IN, USA and ${ }^{2}$ Department of Oncology, Medicine, \& Neurosurgery, Johns Hopkins University School of Medicine, Baltimore, MD, USA

Sir,

In this manuscript, Dr Wong and colleagues observed that patients with recurrent glioblastoma who underwent therapy with tumor treating alternating electrical fields and were on higher doses of dexamethasone had lower T-lymphocyte counts and shorter survival (Wong et al, 2015). The investigators attributed these outcomes entirely to 'global immunosuppression by dexamethasone'. In fact, this patient population receives three therapies that are highly toxic to lymphocytes - glucocorticoids, radiation, and temozolomide. Recent studies have demonstrated that $40 \%$ of patients with newly diagnosed glioblastoma develop grade III and IV lymphopenia with CD4 counts $<200$ cells $\mathrm{mm}^{-3} 2$ months after beginning radiation and temozolomide (Grossman et al, 2011). This profound lymphopenia lasts for over 1 year and on multivariate analysis is independently associated with inferior survival. Treatment-induced lymphopenia has also been studied in other solid tumors that are not treated with dexamethasone or temozolomide; data from these studies strongly point to radiation as the primary causative factor in treatment-induced lymphopenia. These studies reported rates of lymphopenia that were very similar to those seen in glioblastoma and again identified an association between treatment-induced lymphopenia and survival in patients with pancreatic cancer, non-small cell lung cancer, and breast cancer. (Balmanoukian et al, 2012; Campian et al, 2013; Wild et al, 2013; Tang et al, 2014; Afghahi et al, 2015) Two hypotheses have been advanced to explain this phenomenon. The first relates to the inadvertent radiation of circulating lymphocytes (MacLennan and Kay, 1978; Yovino et al, 2013). The second stems from observations that IL-7 levels are inappropriately low in irradiated patients with severe treatment-related lymphopenia (Ellsworth et al, 2014). We agree that immune status is an important prognostic factor in patients with recurrent glioblastoma. 